\title{
Diagnosis of Hypersensitivity Induced by Antituberculosis Drugs
}

\author{
Yuqing Wu, ${ }^{1,2}$ Guangming Xiao, ${ }^{1,2}$ Peilan Zong,, ${ }^{1,2}$ Guoqiang Jiang, ${ }^{1,2}$ Yongmei Liao, ${ }^{1,2}$ \\ Zhou Liu, ${ }^{1,2}$ and Yanhong Zhou ${ }^{10,2}$ \\ ${ }^{1}$ Department of Tuberculosis, Jiangxi Chest Hospital, Nanchang 330006, Jiangxi, China \\ ${ }^{2}$ Jiangxi Clinical Research Center for Infectious Diseases, Nanchang 330006, Jiangxi, China \\ Correspondence should be addressed to Yanhong Zhou; zhouyanhong@jxsxkyy.org.cn
}

Received 11 November 2021; Revised 26 November 2021; Accepted 30 November 2021; Published 20 December 2021

Academic Editor: Kalidoss Rajakani

Copyright (c) 2021 Yuqing Wu et al. This is an open access article distributed under the Creative Commons Attribution License, which permits unrestricted use, distribution, and reproduction in any medium, provided the original work is properly cited.

Objective. To explore the clinical value of the specific plasma cell detection and specific T lymphocyte detection test in diagnosing hypersensitivity caused by antituberculosis drugs. Methods. A total of 266 patients with pulmonary tuberculosis who developed hypersensitivity during the treatment of primary pulmonary tuberculosis in our hospital and 266 patients without hypersensitivity during the treatment of pulmonary tuberculosis in our hospital were selected as the control group. The admission time is from January 2013 to June 2020. The specific plasma cell test and specific T lymphocyte test were used as the criteria to determine which drugs induced hypersensitivity, and the diagnostic value of these two methods in the diagnosis of hypersensitivity induced by four first-line antituberculosis drugs (isoniazid (INH), ethambutol (EMB), rifampicin (RFP), and pyrazinamide (PZA)) was analyzed. Results. The sensitivity of the specific plasma cell test in the diagnosis of hypersensitivity induced by INH, EMB, RFP, and PZA was $63.42 \%, 51.20 \%, 47.81 \%$, and $56.37 \%$, respectively, and the specificity was $95.33 \%, 99.87 \%, 96.52 \%$, and $99.99 \%$, respectively. The sensitivity of the specific Tlymphocyte test in the diagnosis of hypersensitivity induced by INH, EMB, RFP, and PZA was $66.47 \%, 52.88 \%, 49.91 \%$, and 58.54\%, respectively, and the specificity was $97.28 \%, 99.99 \%, 98.38 \%$, and $100.00 \%$, respectively. Conclusion. The specific plasma cell test and specific T lymphocyte test have high specificity in the diagnosis of hypersensitivity caused by antituberculosis drugs, and the specific T lymphocyte test is better than the specific plasma cell test. It is of great significance to guide the clinical application of antituberculosis drugs.

\section{Introduction}

Tuberculosis is one of the infectious diseases that threaten human health [1]. According to WHO statistics, there were 10.4 million new tuberculosis cases worldwide before 2016, with about 1.6 million deaths [2]. China is still one of the 30 countries with high TB burden in the world [3], with about 900000 new TB patients every year, ranking third in the world [4]. At present, the standard treatment regimen consisting of isoniazid (INH), rifampicin (RFP), ethambutol (EMB), and pyrazinamide (PZA) can effectively treat most newly diagnosed tuberculosis patients $[5,6]$. However, these antituberculosis chemical drugs may cause a variety of adverse reactions. Hypersensitivity is one of the common adverse reactions, which is often called drug allergy in clinics [7-9]. Drug hypersensitivity syndrome (DIHS) is a severe allergic reaction, which is characterized by fever, rash, enlarged lymph nodes, eosinophilia, proliferation of atypical lymphocytes in peripheral blood, impaired liver function, or other organ function damage. Its incidence is $1: 10000-1: 1000$, and the case fatality rate is as high as $10 \%[10,11]$. Studies have shown that DIHS caused by antituberculosis drug (ATD) accounts for $13.3 \%$ of all DIHS, ranking third [12]. ATDinduced DIHS has its own characteristics; it is a combination of drugs; it is difficult to determine sensitizing drugs, once the first-line ATD is sensitized; it is difficult to choose alternative treatment, resulting in tuberculosis drug resistance and treatment failure. At present, the commonly used method for the diagnosis of hypersensitivity caused by antituberculosis drugs is the drug lymphocyte stimulation test, which has a high specificity in the diagnosis of patients with hypersensitivity caused by antituberculosis drugs, although the sensitivity is slightly low. However, it is still of great significance to guide the clinical use of drugs. 
According to the Gell-Coombs classification method, allergic reactions are divided into fast hairstyle (I type), cytotoxic (II type), immune complex (IV type), delayed-type hypersensitivity (III type), and allergic reactions caused by antituberculosis drugs mainly for delayed-type hypersensitivity (V type) [13]. Current clinical diagnosis of allergic reactions caused by antituberculosis drugs mainly rely on the drug test (DPT), due to anti-tb drugs are often combined application; however, it can cause allergic reactions or even again exfoliative dermatitis; prolonged hospitalization in the patients not only increase the pain of the patients at the same time but also to a certain extent a blow to treat disease of confidence, hardly conducive to the prevention and control of tuberculosis in China [14]. China has the second largest TB burden in the world [15], How to quickly, safely, and accurately identify drugs that cause hypersensitivity reactions is a problem that clinicians urgently need to solve. The purpose of this study is to explore the sensitivity and specificity of the specific plasma cell test and specific $\mathrm{T}$ lymphocyte test in the diagnosis of antituberculosis druginduced hypersensitivity, so as to lay a foundation for the comprehensive in vitro detection of antituberculosis druginduced hypersensitivity in the future.

\section{Materials and Methods}

\subsection{Study Design and Participants}

2.1.1. General Information. A total of 266 patients who developed hypersensitivity during the treatment of pulmonary tuberculosis in our hospital from January 2013 to June 2020 were selected (group A). 266 patients without hypersensitivity during the treatment of pulmonary tuberculosis in our hospital were selected as the control group (group B). There were 266 patients in group A, aged from 22 to 67 years old, with an average age of $(41.5 \pm 5.3)$ years. 266 patients in group B served as the control group, aged from 18 to 69 years old, with an average age of $(40.9 \pm 6.4)$ years. There was no significant difference in sex $\left(\chi^{2}=0.823\right)$ and age $(t=1.962)$ between the two groups $(P>0.05)$. This study was examined and approved by the ethics committee of our hospital, and all the patients had informed consent.

2.1.2. Inclusion Criteria. (1) It conforms to the relevant diagnostic criteria of newly treated pulmonary tuberculosis in the tuberculosis diagnosis and treatment guidelines of Tuberculosis Branch of Chinese Medical Association; and (2) the clinical manifestation and laboratory examination accorded with the diagnosis of drug-induced hypersensitivity in Clinical Dermatology, which was jointly diagnosed by two specialists.

2.1.3. Exclusion Criteria. (1) Complicated with HIV infection; (2) complicated with chronic kidney disease, diabetes, hematopoietic system diseases, and autoimmune-related diseases; (3) taking antituberculosis treatment while taking other disease treatment drugs; (4) misdiagnosis and misacceptance; and (5) immunosuppressant was used in the process of antiallergic treatment.

\section{Methods}

3.1. Preparation of Experimental Drugs. The single dose of antituberculosis drugs (INH, EMB, RFP, and PZA) taken by the patient was dissolved in $5 \mathrm{ml}$ double distilled water or dimethyl sulfoxide (DMSO) by severe concussion; then, the bacteria were removed by $0.22 \mu \mathrm{m}$ filter and diluted into three concentration gradients $(1: 10,1: 100$, and $1: 1000)$ in the RPMI-1640 culture medium.

3.2. Isolation of PBMC from Peripheral Blood Mononuclear Cells. After the patient was enrolled in the group, $10 \mathrm{ml}$ venous blood was collected for preparation PBMC, $1 \times$ phosphate buffer (PBS) was isolated from heparin anticoagulant blood samples by density gradient centrifugation, resuspended in $2 \mathrm{ml}$ RPMI-1640 culture medium (containing $10 \%$ fetal bovine serum + penicillin streptomycin) and counted, and the cell concentration was adjusted to $1 \times 10^{6} / \mathrm{ml} .1 \times 10^{5} / 100 \mu \mathrm{l}$ PBMC was added to each well of 96-well plate, and then, $5 \mu \mathrm{l}$ of preprepared stimulant drug was added. Negative control holes (drug dissolving medium + culture medium + PBMC), positive control holes (phytohemagglutinin (PHA) $5 \mu \mathrm{g} / \mathrm{ml}+$ culture medium + PBMC), and zeroing holes (only culture medium) were set up, and there were 3 compound holes in each group. At the same time, the anticoagulant blood samples of 1 patient in the control group were randomly selected for the same experiment. The culture plate was placed at $37^{\circ} \mathrm{C}$ and cultured in $5 \% \mathrm{CO}_{2}$ incubator for $60 \mathrm{~h}$, and the cell growth was observed under the inverted microscope.

\subsection{Specific Plasma Cell Detection and Specific T Lymphocyte} Detection. Flow cytometry (BD company FACSCaliburTM) was used to detect specific plasma cells and specific $\mathrm{T}$ lymphocytes. According to the requirements of the reagent instructions, $20 \mu \mathrm{L}$ of TriTESTCD4/CD8/CD3 reagent and $50 \mu \mathrm{L}$ of peripheral anticoagulant were added into the counter tube to mix evenly. After incubating $15 \mathrm{~min}$ without light, $450 \mathrm{ml}$ of hemolytic agent was added, mixed, and incubated without light for $15 \mathrm{~min}$ and then detected on the computer.

3.4. Determination of Drugs Leading to Hypersensitivity. After the symptoms of hypersensitivity were relieved after drug withdrawal and the results of specific plasma cell test and specific T lymphocyte test were determined, DPT (the gold standard for clinical judgment of which drug caused hypersensitivity) was performed. The interval between each drug was 3-4 d. Once DPT was positive during the test period, it could be determined as the hypersensitivity of the drug.

3.5. Statistical Method. SPSS25.0 statistical software was used for data analysis. The measurement data were expressed by $(x \pm s)$, the two groups were compared with two 
independent samples $t$-test, and the counting data were compared with the $\chi^{2}$ test. The difference was statistically significant $(P<0.05)$.

\section{Results}

4.1. Diagnostic Value of Specific Plasma Cell Detection and Specific T Lymphocyte Detection Test in Antituberculosis DrugInduced Hypersensitivity. A total of 272 cases of antituberculosis drug-induced hypersensitivity were detected by DPT ( 4 cases were caused by EMB, PZA, and RFP and 2 cases were induced by EMB and RFP). A total of 5 cases of hypersensitivity induced by two antituberculosis drugs were detected by the specific plasma cell test and specific T lymphocyte test ( 3 cases by EMB and PZA and 2 cases by INH and RFP).

The sensitivity of the specific plasma cell test in the diagnosis of hypersensitivity induced by INH, EMB, RFP, and PZA was $63.42 \%, 51.20 \%, 47.81 \%$, and $56.37 \%$, respectively, and the specificity was $95.33 \%, 99.87 \%, 96.52 \%$, and $99.99 \%$, respectively (Tables $1-4$ ).

The sensitivity of the specific $\mathrm{T}$ lymphocyte test in the diagnosis of hypersensitivity induced by INH, EMB, RFP, and PZA was $66.47 \%, 52.88 \%, 49.91 \%$, and $58.54 \%$, respectively, and the specificity was $97.28 \%, 99.99 \%, 98.38 \%$, and $100.00 \%$, respectively (Tables 5-8).

\section{Discussion}

Hypersensitivity reaction is one of the most common adverse reactions of antituberculosis drugs at present, and the clinical symptoms of DIHS caused by ATD are various $[16,17]$, including the following typical characteristics: (1) delayed drug anaphylaxis, that is, adverse reactions occur within 2 weeks to 3 months after the use of ATD; (2) clinical manifestations and laboratory tests are similar to virus infection; and (3) symptoms persist or worsen after withdrawal of related drugs. Involvement of internal organs is a prominent feature of DIHS. Studies by Husain et al. [18] have shown that drug-induced DIHS may sometimes involve specific organs, such as penicillins, allopurinol, and antiepileptic drugs. ATD is prone to liver injury, including drug direct toxicity and immune-mediated liver injury, that is, hypersensitive liver injury, liver injury caused by lipid peroxidation [19]. In the reported DIHS caused by ATD, visceral organs are involved extensively and severely, and there is no obvious organ specificity, which can lead to liver damage, polymyositis, myocarditis, pneumonia, and acute renal failure $[20,21]$. Even in hemophagocytic syndrome antibiotics are ineffective when DIHS is involved in the lungs, but lung lesions are absorbed after glucocorticoid treatment. The pathological changes of the skin are prominent; different from drug eruptions such as severe pleomorphic erythema and toxic epidermal necrolysis, urticaria and macular papules are the most common [22].

The pathogenesis may be related to virus activation, abnormal immune response, and genetic susceptibility factors. It can be explained that in susceptible individuals [23], with certain (HLA) alleles of human leukocyte antigen,
TABLE 1: Diagnostic value of the specific plasma cell test in INHinduced hypersensitivity.

\begin{tabular}{lccc}
\hline \multirow{2}{*}{ Specific plasma cell test } & \multicolumn{2}{c}{ DPT } & Total \\
& Positive & Negative & \\
\hline Positive & 88 & 15 & 103 \\
Negative & 40 & 389 & 431 \\
Total & 128 & 404 & 532 \\
\hline
\end{tabular}

taking related drugs for a certain time and dose, due to the deficiency of drug metabolic enzymes and the accumulation of toxic metabolites, lead to the formation of new antigens between semiantigens, intermediate reaction metabolites, and tissue macromolecules, and the costimulatory signal pathway is induced by antigen presenting cells; thus, the potential virus is activated and cloned T cells are expanded. $\mathrm{T}$ cells produce cytokines, infiltrate into the skin and other organ functions, and increase the number of eosinophils in peripheral blood and tissue [24]. Some studies have pointed out that $12 \mathrm{H}-\mathrm{R}-\mathrm{Z}-\mathrm{E}$ regimen can significantly provide the success rate of treatment, greatly reduce the loss of follow-up rate of patients, effectively reduce hypersensitivity reactions, and help to reduce other adverse drug reactions and reduce the mortality of patients. The most effective way to deal with drug hypersensitivity is to temporarily suspend treatment and wait for the patient's hypersensitivity to recover to offspring with other therapeutic drugs with different chemical structures. This will not only prolong the length of stay of patients but also aggravate the pain of patients, undermine patients' confidence in treating the disease, and even cause patients to give up treatment, which is not conducive to the prevention and control of tuberculosis in our country. Therefore, to find a rapid, accurate, and safe detection method of antituberculosis drugs that lead to hypersensitivity is the common direction of tuberculosis clinical staff.

This study analyzed the diagnostic value of hypersensitivity caused by four first-line antituberculosis drugs (INH, EMB, RFP, and PZA) with specific plasma cell detection and specific $\mathrm{T}$ lymphocyte detection. The results showed that the specific plasma cell detection and specific $\mathrm{T}$ lymphocyte detection test has certain significance in the diagnosis of hypersensitivity caused by antituberculosis drugs. It is mainly characterized by high specificity, but the sensitivity is generally low. Consider the following possible reasons. (1) Drugs: the pathological mechanism of hypersensitivity reactions is complicated, and T lymphocyte immunity cannot fully cover the causes of hypersensitivity reactions caused by various drugs; (2) the patients in this study stay in the hospital within a short period of time after the rash occurs. Take venous blood, considering the influence of the drug washout period. In the future, a variety of in vitro detection methods will be combined in different periods after the hypersensitivity reaction occurs in patients, and the sensitivity is expected to be improved. This study found that the sensitivity and specificity of the specific T lymphocyte test are higher than that of the specific plasma cell test (the sensitivity of the specific plasma cell test to diagnose hypersensitivity caused by INH, EMB, RFP, and PZA is 
TABLE 2: Diagnostic value of the specific plasma cell test in EMBinduced hypersensitivity.

\begin{tabular}{|c|c|c|c|}
\hline \multirow{2}{*}{ Specific plasma cell test } & \multicolumn{2}{|c|}{ DPT } & \multirow{2}{*}{ Total } \\
\hline & Positive & Negative & \\
\hline Positive & 70 & 28 & 98 \\
\hline Negative & 84 & 350 & 434 \\
\hline Total & 154 & 378 & 532 \\
\hline
\end{tabular}

TABle 3: Diagnostic value of the specific plasma cell test in RFPinduced hypersensitivity.

\begin{tabular}{lccc}
\hline \multirow{2}{*}{ Specific plasma cell test } & \multicolumn{2}{c}{ DPT } & Total \\
& Positive & Negative & \\
\hline Positive & 65 & 30 & 95 \\
Negative & 95 & 342 & 437 \\
Total & 160 & 372 & 532 \\
\hline
\end{tabular}

TABle 4: Diagnostic value of the specific plasma cell test in PZAinduced hypersensitivity.

\begin{tabular}{lccc}
\hline \multirow{2}{*}{ Specific plasma cell test } & \multicolumn{2}{c}{ DPT } & Total \\
& Positive & Negative & \\
\hline Positive & 77 & 20 & 97 \\
Negative & 73 & 362 & 435 \\
Total & 150 & 382 & 532 \\
\hline
\end{tabular}

TABle 5: The diagnostic value of the specific $\mathrm{T}$ lymphocyte test in INH-induced hypersensitivity.

\begin{tabular}{lccc}
\hline \multirow{2}{*}{ Specific T lymphocyte test } & \multicolumn{2}{c}{ DPT } & Total \\
& Positive & Negative & \\
\hline Positive & 94 & 13 & 107 \\
Negative & 28 & 397 & 425 \\
Total & 122 & 410 & 532 \\
\hline
\end{tabular}

TABLE 6: Diagnostic value of the specific T lymphocyte test in EMBinduced hypersensitivity.

\begin{tabular}{lccc}
\hline \multirow{2}{*}{ Specific T lymphocyte test } & \multicolumn{2}{c}{ DPT } & Total \\
& Positive & Negative & \\
\hline Positive & 72 & 30 & 102 \\
Negative & 86 & 344 & 430 \\
Total & 158 & 374 & 532 \\
\hline
\end{tabular}

TABLe 7: Diagnostic value of the specific T lymphocyte test in RFPinduced hypersensitivity.

\begin{tabular}{lccc}
\hline \multirow{2}{*}{ Specific T lymphocyte test } & \multicolumn{2}{c}{ DPT } & Total \\
& Positive & Negative & \\
\hline Positive & 60 & 34 & 94 \\
Negative & 103 & 335 & 438 \\
Total & 163 & 369 & 532 \\
\hline
\end{tabular}

$63.42 \%, 51.20 \%, 47.81 \%$, and $56.37 \%$, respectively, with specificities of $95.33 \%, 99.87 \%, 96.52 \%$, and $99.99 \%$, respectively. The sensitivity of specific T lymphocyte detection
TABLE 8: The diagnostic value of the specific $T$ lymphocyte test in PZA-induced hypersensitivity.

\begin{tabular}{lccc}
\hline \multirow{2}{*}{ Specific T lymphocyte test } & \multicolumn{2}{c}{ DPT } & Total \\
& Positive & Negative & \\
\hline Positive & 79 & 34 & 113 \\
Negative & 70 & 349 & 419 \\
Total & 149 & 383 & 532 \\
\hline
\end{tabular}

tests to diagnose hypersensitivity caused by INH, EMB, RFP, and PZA are, respectively, $66.47 \%, 52.88 \%, 49.91 \%$, and $58.54 \%$ and the specificities are, respectively, $97.28 \%$, $99.99 \%, 98.38 \%$, and $100.00 \%$, but this article did not compare the correlation and difference, and this research experiment still needs more. This is further confirmed by a large research population.

There are many adverse reactions of ATD. For severe adverse drug reactions such as DIHS, more attention should be paid to the study of susceptible genes, specific viruses, cytokines, and new biomarkers. Attention should be paid to the adverse reactions of glucocorticoids in the course of treatment. Considering the risk of the drug stimulation test, more safe and effective laboratory examination methods are needed. To sum up, the specific plasma cell test and specific $\mathrm{T}$ lymphocyte test have high specificity in the diagnosis of antituberculosis drug-induced hypersensitivity; although the sensitivity is slightly lower, but it is still of great significance in guiding clinical drug use.

\section{Data Availability}

The analyzed datasets generated during the study are available from the corresponding author upon request.

\section{Conflicts of Interest}

The authors declare that there are no conflicts of interest.

\section{Acknowledgments}

This study was supported by the Key R\&D Program of Jiangxi Provincial Department of Science and Technology (20192BBGL70011) and National Natural Science Foundation of China Regional Project (8196003).

\section{References}

[1] H. Y. Jung, S. Park, B. Shin et al., "Prevalence and clinical features of drug reactions with eosinophilia and systemic symptoms syndrome caused by antituberculosis drugs: a retrospective cohort study," Allergy, Asthma \& Immunology Research, vol. 11, no. 1, pp. 90-103, 2019.

[2] J. Whritenour, M. Ko, Q. Zong et al., "Development of a modified lymphocyte transformation test for diagnosing drug-induced liver injury associated with an adaptive immune response," Journal of Immunotoxicology, vol. 14, no. 1, pp. 31-38, 2017.

[3] J. Klaewsongkram, C. Sukasem, P. Thantiworasit et al., "Analysis of HLA-B allelic variation and IFN- $\gamma$ ELISpot responses in patients with severe cutaneous adverse reactions 
associated with drugs," The Journal of Allergy and Clinical Immunology: In Practice, vol. 7, no. 1, pp. 219-227, 2019.

[4] W. W. Yew, K. C. Chang, and D. P. Chan, "Oxidative stress and first-line antituberculosis drug-induced hepatotoxicity," Antimicrobial Agents and Chemotherapy, vol. 62, no. 8, 2018.

[5] G. K. Buhari, M. Keren, A. B. Dursun et al., "Immediate-type hypersensitivity reactions due to antituberculosis drugs: a successful readministration protocol," Annals of Allergy, Asthma, \& Immunology, vol. 115, no. 1, pp. 39-44, 2015.

[6] H. Kim, E. S. Bang, S.-K. Lim, and J. M. Lee, "DRESS syndrome and acute generalized exanthematous pustulosis induced by antituberculosis medications and moxifloxacin: case report," International Journal of Clinical Pharmacology and Therapeutics, vol. 54, no. 10, pp. 808-815, 2016.

[7] R. Rakotoarimanana, O. H. Rakotonirainy, O. Razanamparany, T. Raonivelo, and S. Ralandison, "Difficultés diagnostiques d'un exanthème fébrile d'étiologies différentes chez une même patiente tuberculeuse à Madagascar," Bulletin de la Société de Pathologie Exotique, vol. 105, no. 5, pp. 349-352, 2012.

[8] S.-N. Zhang, Q.-X. He, N.-B. Yang, S.-L. Ni, and M.-Q. Lu, "Isoniazid-induced drug rash with eosinophilia and systemic symptoms (DRESS) syndrome presenting as acute eosinophilic myocarditis," Internal Medicine, vol. 54, no. 10, pp. 1227-1230, 2015.

[9] O. Katsube, M. Anzai, Y. Nomura et al., "[A case of druginduced hypersensitivity syndrome caused by levofloxacin used for treating pulmonary tuberculosis]," Kekkaku, vol. 89, no. 2, pp. 51-56, 2014

[10] G. Avcu, G. Sensoy, M. H. Çeliksoy et al., "Cutaneous leukocytoclastic vasculitis associated with anti-tuberculosis drugs," Pediatrics International, vol. 57, no. 1, pp. 155-157, 2015.

[11] D. Yee, C. Valiquette, M. Pelletier, I. Parisien, I. Rocher, and D. Menzies, "Incidence of serious side effects from first-line antituberculosis drugs among patients treated for active tuberculosis," American Journal of Respiratory and Critical Care Medicine, vol. 167, no. 11, pp. 1472-1477, 2003.

[12] L. Wang and L.-F. Li, "Difficult clinical management of antituberculosis DRESS syndrome complicated by MRSA infection," Medicine, vol. 96, no. 11, Article ID e6346, 2017.

[13] E. J. Forget and D. Menzies, "Adverse reactions to first-line antituberculosis drugs," Expert Opinion on Drug Safety, vol. 5, no. 2, pp. 231-249, 2006.

[14] H. Devarbhavi, "An update on drug-induced liver injury," Journal of Clinical and Experimental Hepatology, vol. 2, no. 3, pp. 247-259, 2012.

[15] A. Sekine, T. Saito, S. Ito et al., "Two cases of tuberculosis with multiple drug hypersensitivity after drug-induced hypersensitivity syndrome," Respiratory Investigation, vol. 50, no. 2, pp. 70-75, 2012.

[16] S. Rezakovic, Z. Pastar, and K. Kostovic, "Cutaneous adverse drug reactions caused by antituberculosis drugs," Inflammation and Allergy - Drug Targets, vol. 13, no. 4, pp. 241-248, 2014.

[17] J. Wang, M. Bwayi, R. R. F. Gee, and T. Chen, "PXR-mediated idiosyncratic drug-induced liver injury: mechanistic insights and targeting approaches," Expert Opinion on Drug Metabolism and Toxicology, vol. 16, no. 8, pp. 711-722, 2020.

[18] Z. Husain, B. Y. Reddy, and R. A. Schwartz, "DRESS syndrome: part I. clinical perspectives," Journal of the American Academy of Dermatology, vol. 68, no. 5, pp. 691-693, 2013.

[19] A. Coster, O. Aerts, A. Herman et al., "Drug reaction with eosinophilia and systemic symptoms (DRESS) syndrome caused by first-line antituberculosis drugs: two case reports and a review of the literature," Contact Dermatitis, vol. 81, no. 5, pp. 325-331, 2019.

[20] T. Shiohara and Y. Kano, "Drug reaction with eosinophilia and systemic symptoms (DRESS): incidence, pathogenesis and management," Expert Opinion on Drug Safety, vol. 16, no. 2, pp. 139-147, 2017.

[21] H. Watanabe, "Recent advances in drug-induced hypersensitivity syndrome/drug reaction with eosinophilia and systemic symptoms," Journal of Immunol Res, vol. 18, Article ID 5163129, 2018.

[22] M. Garcia-Cortes, M. Robles-Diaz, C. Stephens, A. OrtegaAlonso, M. I. Lucena, and R. J. Andrade, "Drug induced liver injury: an update," Archives of Toxicology, vol. 94, no. 10, pp. 3381-3407, 2020.

[23] A. T. Castro, M. Mendes, S. Freitas, and P. C Roxo, "Incidence and risk factors of major toxicity associated to first-line antituberculosis drugs for latent and active tuberculosis during a period of 10 years," Revista Portuguesa de Pneumologia, vol. 21, no. 3, pp. 144-150, 2015.

[24] S.-H. Kim, S.-H. Kim, H. J. Yoon et al., "GSTT1 and GSTM1 null mutations and adverse reactions induced by antituberculosis drugs in Koreans," Tuberculosis, vol. 90, no. 1, pp. 39-43, 2010. 